\title{
Análisis estilístico de los conjuntos del «Canjorro de Peñarrubia», «Doña Clotilde», «Abrigo del tío Campano» y «Selva Pascuala»
}

\author{
Manuel lópez Payer \\ MIGUEL SORIA LERMA
}

\section{INTRODUCCIÓN}

Uno de los aspectos en los que la investigación del arte rupestre adolecía de un mayor estancamiento, era el relativo al análisis estilístico. Con el fin de resolver este problema, elaboramos hace poco tiempo un método analítico basado en la aplicación de una escala de naturalismo y en el estudio y cuantificación de los convencionalismos formales de las figuras (LOPEZ PAYER y SORIA LERMA, 1988, págs. 207-219).

Una de las primeras aplicaciones de nuestro modelo de investigación, fue realizada sobre todas las figuras de cérvidos y cápridos seminaturalistas y semiesquemáticos del arte rupestre postpaleolítico de los núcleos más orientales de Andalucía (LOPEz PAYER y SORIA LeRMA, 1991, en prensa). En una primera valoración de los resultados, el metodo empleado se ha mostrado como una herramienta totalmente válida en orden al establecimiento de fases y estilos, objetivamente constatados, y de gran efectividad para analizar toda una serie de figuras, cuyo grado de naturalismo y cuya morfología han sido la base de multitud de hipótesis contradictorias.

Dentro de este proceso, ya iniciado, hemos considerado realmente interesante abordar el estudio de varios grupos que poseen como carac- 
terística común la presencia de escenas de domesticación de caza de équidos vivos, cuyas figuras manifiestan a su vez un acusado grado de naturalismo. Nos referimos a los conjuntos del "Canjorro de Peñarrubia" en Sierra Morena, a los de "Doña Clotilde" y "Abrigo del Tio Campano" en Albarracín (Teruel), y al de "Selva Pascuala" en Villar del Humo (Cuenca).

Casi todos estos conjuntos eran ya muy conocidos desde hace varias décadas, siendo, por sus especiales características, objeto de diversas interpretaciones, especialmente en orden a su posición con respecto a las diferentes fases de desarrollo de los estilos Levantino y/o Esquemático. Aunque la mayoría de los investigadores habian advertido su grado medio de naturalismo, calificando sus figuras como seminaturalistas a pesar de lanzar sus críticas sobre este tipo de términos, no es menos cierto que también habian señalado que su morfología no era la típica de los citados estilos; a pesar de ello, no se ha dudado, en razón de su situación geográfica, en incluirlos en las monografías dedicadas al Estilo Esquemático, como es el caso del "Canjorro de Peñarrubia", o en las del Estilo Levantino, caso del resto de los conjuntos. Es evidente, por lo tanto, que se hace necesaria una clarificación estilística de estos conjuntos, sin partir a priori con la idea de su inclusión forzosa en uno u otro estilo, y hacer una valoración de los conjuntos en si mismos, de su grado de naturalismo y de sus convencionalismos formales. Sólo así delimitaremos su estilo y obtendremos una sólida base para intentar posteriormente buscar una experiencia a sus especiales características.

\section{EL “CANJORRO DE PEÑARRUBIA»}

Este yacimiento se encuentra en la vertiente meridional de Sierra Morena Oriental, en el núcleo «Los Guindos-El Centenillo» (Baños de la Encina, Jaén). Fué estudiado por primera vez en la campaña que $H$. Breuil y J. Cabré efectuaron por esta zona del 1 al 27 de mayo de 1913. Desde la publicación de BreUIL (1933, vol. III, págs. 6, 49-54; láms. XVIII, $\mathrm{XIX} 1$ y $\mathrm{XX}$ ) es, posiblemente, el yacimiento más divulgado de toda Sierra Morena.

Después de Breuil han sido muchos los investigadores que han estudiado este conjunto, aunque sólo unos pocos lo han hecho de forma directa. Tal es el caso del profesor E. Hernández-Pacheco, que incluyó 


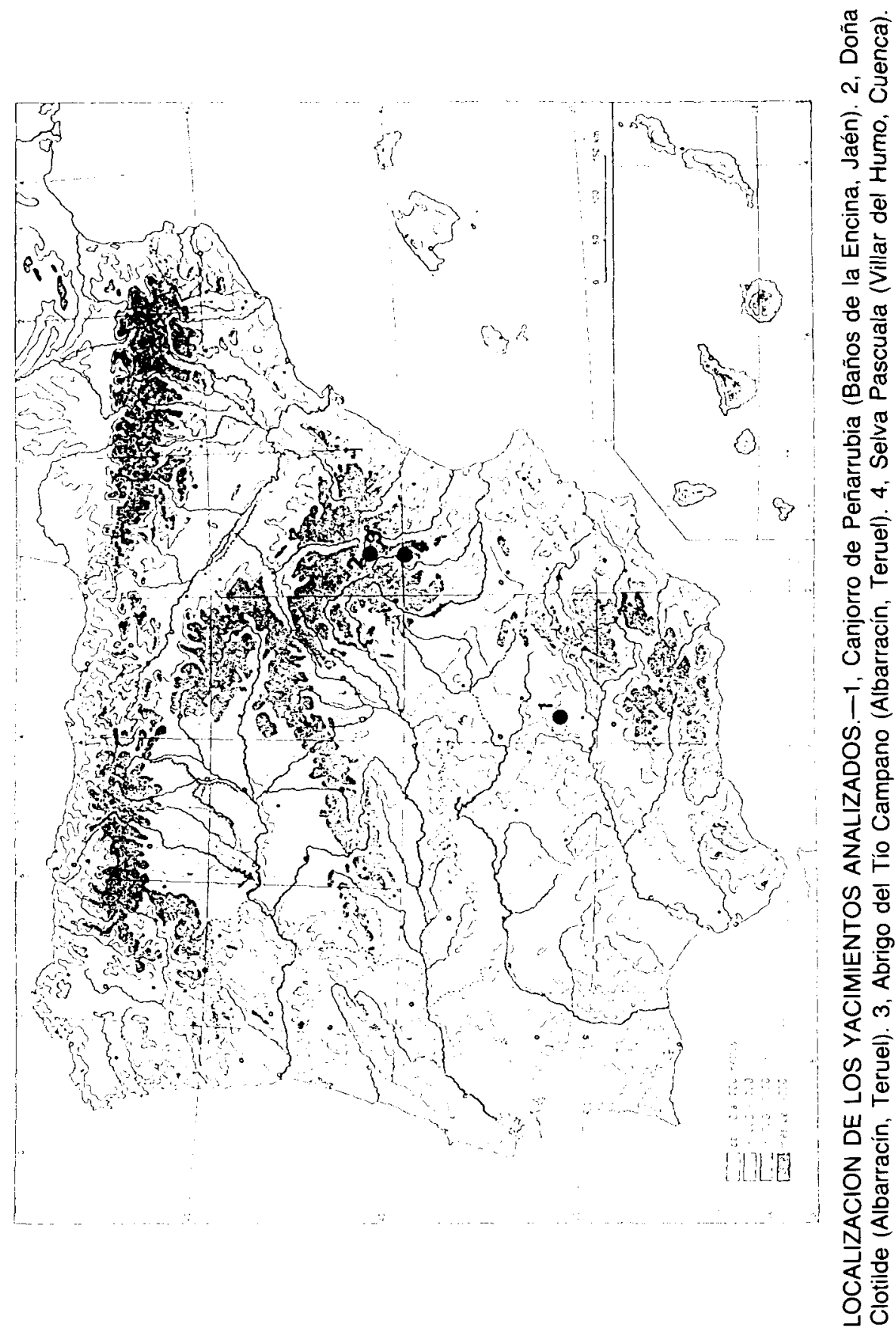


su estudio dentro de un grupo de conjuntos de estilo similar (HERnÁNDEZPACHECO, 1959, págs. 341-527).

Posteriormente, el francés Guy Tamain realizó dos estudios monográficos. En el primero de ellos, creyéndolo inédito, lo bautizó con el nombre de "Barranco de la Moneda" (TAMAIN, 1962, págs. 220-229), error que rectificó en una segunda publicación (TAMAIN, 1963, págs. 828837). En ambos trabajos faltaban gran cantidad de figuras.

Por último, nosotros efectuamos una revisión reciente en la que presentamos un calco que reflejaba el estado actual del conjunto y haciamos una serie de observaciones estilísticas (LOPEZ PAYER y SORIA, 1988, págs. 124-127 y 439-446).

El especial interés que ha suscitado este yacimiento, ha sido motivado por la diversidad tipológica y estilística de sus figuras, entre las que se pueden apreciar dos arqueros, varios antropomorfos esquemáticos y una serie de figuras humanas con ciertos detalles anatómicos, que aparecen formando parejas o sujetando a équipos por la brida. El grado de naturalismo de las figuras citadas en contraste con las que no poseen esta cualidad, ha servido de base para el establecimiento de dos fases estilísticas (BREUIL, 1933, vol III, págs. 49-54), opinión que comparten la mayoría de los investigadores, excepto Tamain, que señala tres estilos distintos: uno naturalista, representado por las parejas humanas y las escenas de domesticación; otro naturalista-residual, representado por los arqueros; y otro esquemático, representado por el resto de los antropomortos (TAMAin, 1963, págs. 835-836).

Ante la diversidad de opiniones y la disparidad de términos, se hace necesaria la objetivación del estudio estilístico. Esta es la razón por la que hemos aplicado nuestro método analítico obteniendo los siguientes resultados:

a) Resultados de la aplicación de la Escala de Naturalismo:

1. Antropomorfo ....4 puntos .....Estilo seminaturalista

2. Zoomorfo .......4,75 puntos .....Estilo seminaturalista

3. Antropomorfo .....3,5 puntos .....Estilo semiesquemático

4. Zoomorfo ........2,75 puntos .....Estilo semiesquemático

b) Análisis de los convencionalismos formales:

Antropomorfos: Dentro de este tipo de figuras, distinguimos en primer lugar una serie de parejas y de figuras humanas asociadas a équidos, que arrojan una puntuación similar en la escala de naturalismo (3,5-4 


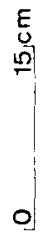
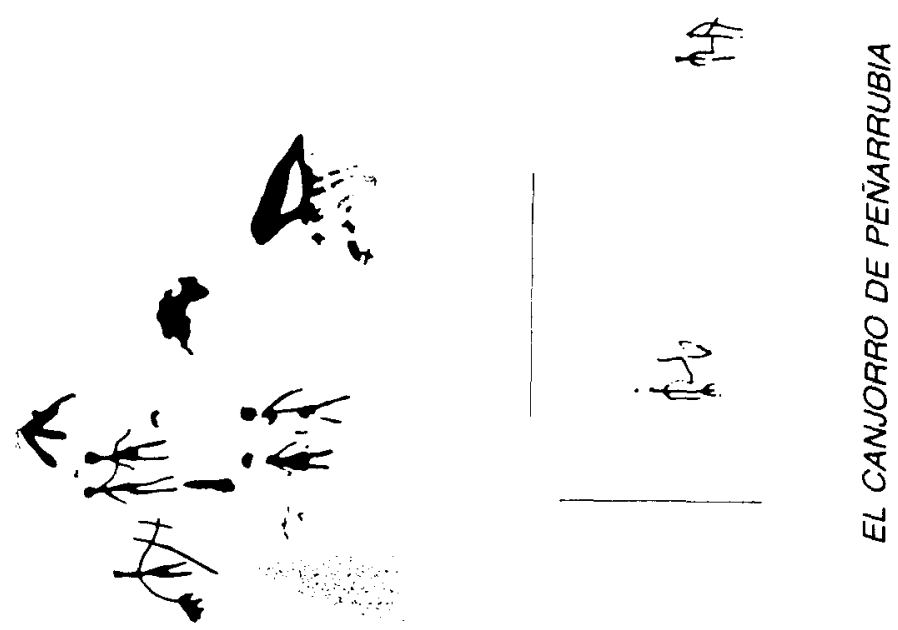

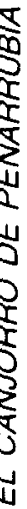

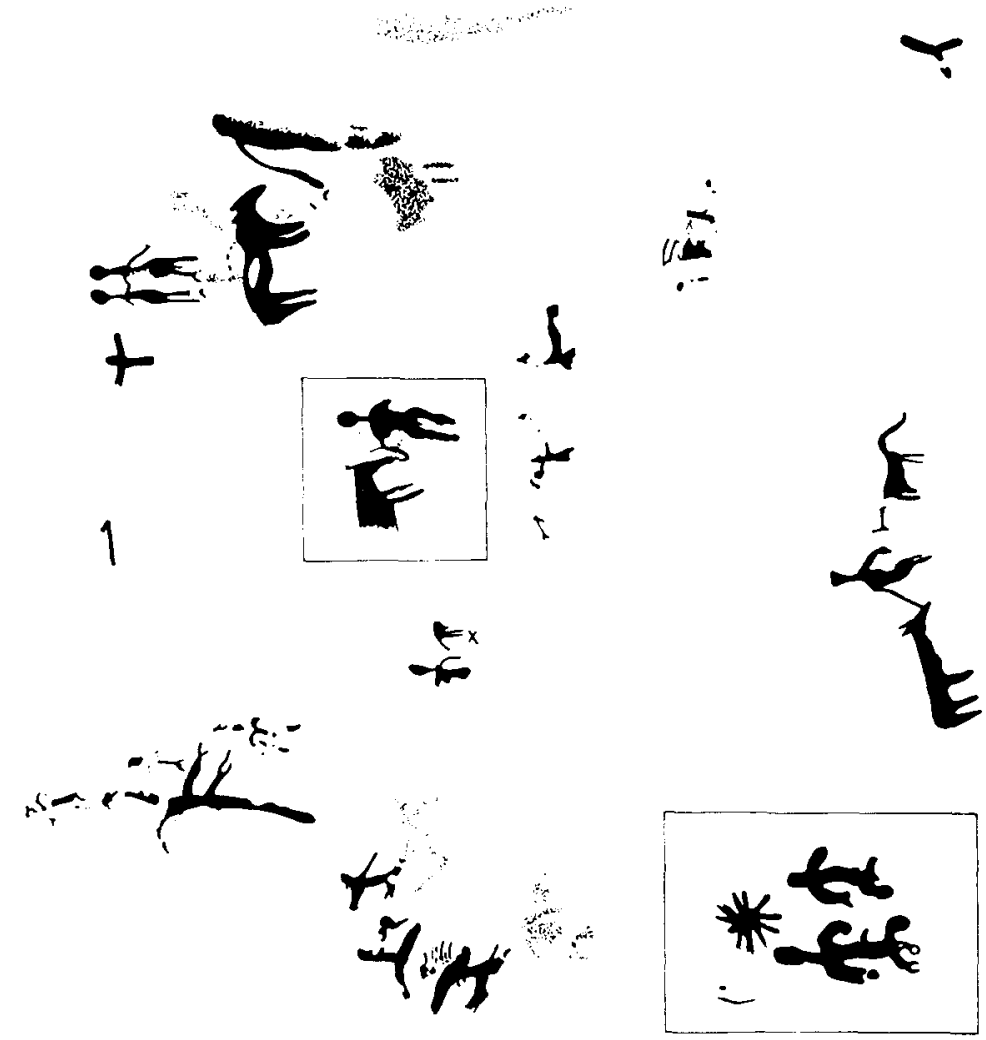


puntos) y que poseen una serie de características homogéneas que se pueden resumir, salvo mínimas excepciones, de la siguiente forma:

- Cabeza generalmente redonda y sin detalles.

- El cuello está generalmente representado.

- Tórax estrecho en las parejas y ancho en las figuras asociadas a équidos.

- Abdomen abultado.

- Extremidades superiores de fino trazado, arqueadas hacia abajo y sin expresión de mano o dedos.

- Extremidades inferiores delgadas y rígidas en las parejas y algo abultadas en las asociadas a équidos. Sólo una figura posee representado un pie.

Excepto una figura humana semiesquemática que se encontraba formando pareja con otra y ambas se asociaban a un "sol», las cuales han desaparecido, el resto de los antropomorfos son esquemáticos, pudiendo éstos subdividirse en dos grupos: el primero estaria constituido por los arqueros de la entrada, caracterizados por su fino trazado y por una cabeza aplanada que puede indicar la presencia de un determinado peinado, tocado o sombrero. Uno de los antropomorfos asociados a équidos posee un convencionalismo similar. El segundo grupo lo formarian el resto de los antropomorfos, mal conservados en general, siendo el de la parte superior el más representativo. Sus rasgos con los que definen al subtipo “típico simple».

Zoomorfos: Se trata de un grupo de figuras, algunas de ellas incompletas, con desigual puntuación en la escala de naturalismo $(2,75$ y 4,75 puntos) pero que poseen los siguientes rasgos comunes:

- Cabeza con cierta apariencia real y representación de las orejas.

- Cuello con la parte superior convexa o recta y la inferior cóncava o algo sinuosa.

- Todos ellos se unen a un antropomorfo por un trazo fino que representa la brida.

- Extremidades rígidas sin expresión de pezuñas ni articulaciones.

En consecuencia, el análisis estilístico desvela la presencia de tres bloques de figuras: El primero estaria constituido generalmente por figu- 

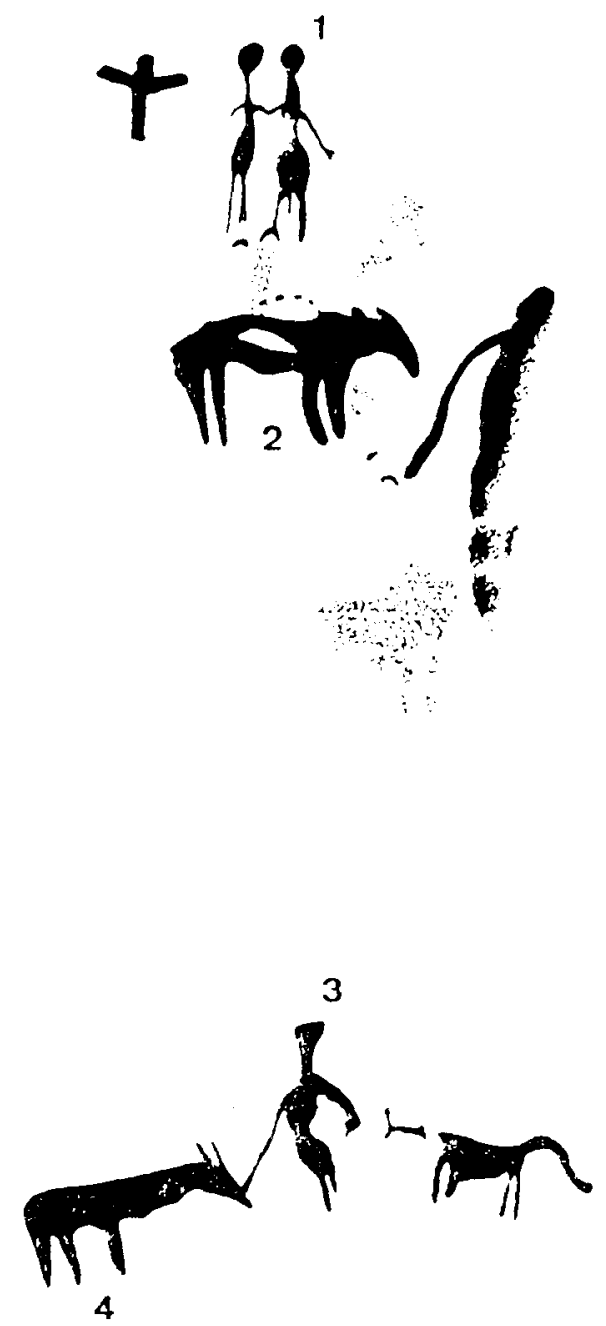

FIGURAS ANALIZADAS DEL CANJORRO DE PEÑARRUBIA 
ras seminaturalistas (parejas y asociaciones de antropomorfos y équidos), el segundo bloque estaría formado por las figuras esquemáticas de los arqueros, y por último, el tercer bloque lo formarían el resto de los antropomorfos.

Como se puede observar, este análisis viene a apoyar en parte a las observaciones efectuadas por Tamain, aunque no coincidimos en la apreciación del citado "naturalismo residual" de los arqueros, calificativo impreciso y vago que no debe adjudicarse a estas figuras, que poseen convencionalismos formales diferentes pero que no dejan de ser esquemáticas.

\section{LA “CUEVA DE DOÑA CLOTILDE"}

Este yacimiento, ubicado en el núcleo de Albarracín (Teruel), fue investigado por primera vez por el profesor ALMAGRO BASCH (1944, págs. 1-38), siendo su calco el más difundido y utilizado por la mayoría de los investigadores.

De este conjunto fue realizada una revisión por $F$. Piñón, que presentó un nuevo calco del mismo (PIÑON, 1982, págs. 105-122). Este nuevo calco difiere en parte del primero, lo que es explicable por la dificultad de su realización, al poseer muchas figuras una coloración similar a la roca soporte.

Componen este conjunto una cuarentena de figuras de diversa tipología y estilo, destacando 24 antropomorfos, 13 zoomorfos, un arboriforme y algunos signos más.

Los antropomorfos se dividen a su vez en varios grupos: cuatro de ellos tienen aspecto globuloso, tres son del tipo "ancoriforme" y el resto responden a una morfología "filiforme", con un cuerpo alargado y un tocado aplanado, algunos de los cuales llevan un arco, siendo los de la izquierda más finos y con menor riqueza de detalles que el resto.

Entre los 13 zoomorfos que distingue F. Piñón, destacan un zorro invertido (muerto), 3 équidos y varios más de tipo indeterminado.

La escena más llamativa es aquella en la que aparece un antropomorfo globuloso con varios trazos a modo de varas en la misma mano que sujeta por la brida a un équido. Esta escena, como vemos, es muy similar a las ya estudiadas del "Canjorro de Peñarrubia", y podemos 

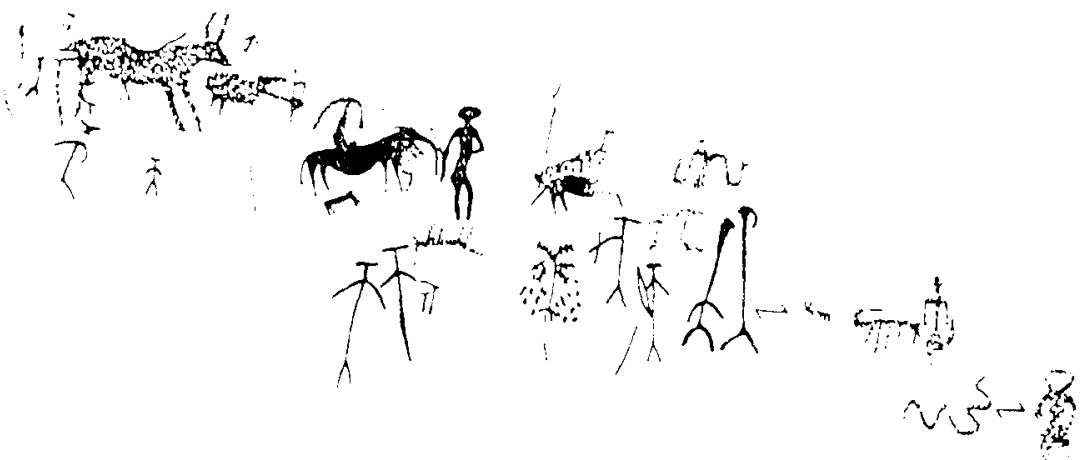

CUEVA DE DOÑA CLOTILDE

(Según M. Almagro Basch)

considerarla representativa del resto de los antropomorfos y zoomorfos que poseen un cierto naturalismo, por lo que hemos procedido a analizarla obteniendo los siguientes resultados:

a) Resultados de la aplicación de la Escala de Naturalismo:

1. Antropomorfo ....4 puntos ........Estilo seminaturalista.

2. Zoomorfo ........5 puntos. ........Estilo seminaturalista.

b) Análisis de los convencionalismos formales:

Antropomorfos: Entre los rasgos más comunes y característicos de estas figuras de aspecto globuloso, podemos señalar los siguientes:

- La figura analizada posee cabeza semicircular (según Piñón) o redondeada como el resto (según Almagro).

- Las extreminades superiores son delgadas y arqueadas hacia abajo, excepto la figura principal que las posee flexionadas por el codo. No aparecen las manos ni los dedos.

- Extreminades inferiores rígidas sin expresión de los pies.

- Ligera indicación de la cintura mediante un estrechamiento que separa el tórax del abdomen, los cuales aparecen algo más abultados. 


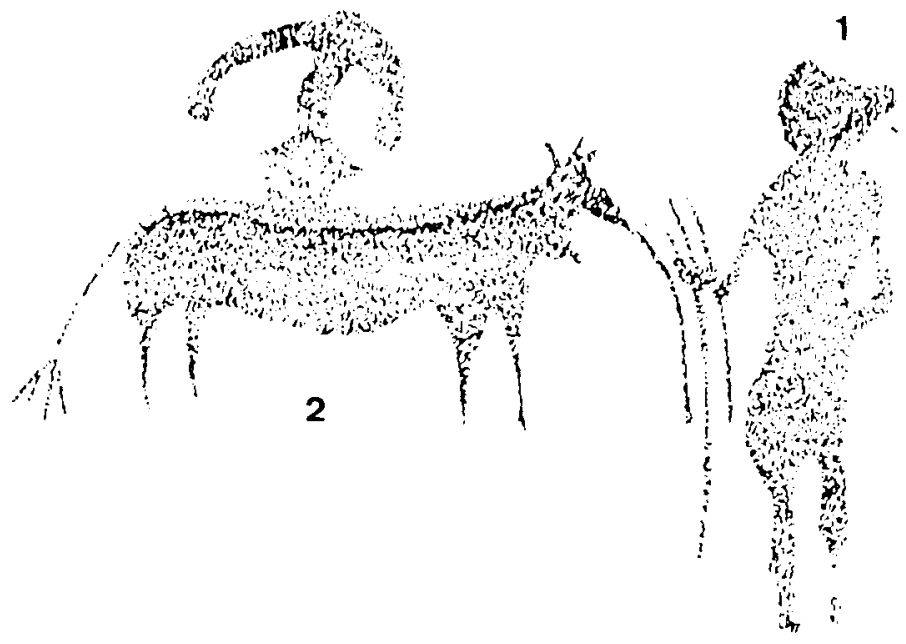

CUEVA DE DONA CLOTILDE

(Según F. Piñon)

Zoomorfos: A pesar de que, por tratarse de animales diferentes, poseen rasgos diferentes, podemos deducir algunas caracteristicas comunes, como son:

- Cabeza de aspecto real con expresión de orejas y/o cuernos.

- Cuello convergente hacia la cabeza.

- Linea cervico-dorsal cóncava.

- Rabo largo.

- Extremidades rígidas sin expresar detalles como las pezuñas o las articulaciones.

- Ronzal fino en prolongación del hocico.

Entre las figuras no analizadas, algunos de los antropomorfos de fino trazado poseen, según el calco de F. PIÑón (1982, pág. 217), ciertos detalles anatómicos que podrian situarlos dentro de un estilo semiesquemático; sin embargo, debido a su difusa definición en el citado calco y a que en el calco del profesor Almagro no aparecen esos detalles, no le hemos aplicado nuestra escala de naturalismo. 
Globalmente, F. Piñón distinguió, basándose en las superposiciones $y$ en diferentes detalles, un total de seis fases compositivas (PINON, 1982, págs. 118-120). Por nuestra parte, basándonos en los diferentes tipos y estilo de los antropomorfos, pensamos que se puede reducir a tres, las cuales pueden estar respectivamente representadas por cada uno de los tres tipos de antropomorfos: "ancoriformes", antropomorfos de trazo fino, cañon alargado y cabeza o tocado aplanado, y antropomorfos globulosos seminaturalistas asociados a zoomorfos del mismo estilo.

La posición cronológica de cada una de estas fases no se puede dilucidar fácilmente, pues aunque en el calco de F. Piñón nos muestra que la escena de domesticación se superpone a un "ancoriforme" y a un zoomorto de color rojo más claro (PINON, 1983, pág. 379), esta circunstancia no está tan clara en el dibujo del profesor Almagro.

\section{EL «ABRIGO DEL TIOO CAMPANO»}

El estudio de este abrigo, perteneciente como el anterior al núcleo de Albarracin (Teruel), fue realizado por F. PINON (1982, págs. 203-207, y 1983, págs. 371-384), que distinguió en él un total de cinco figuras muy deterioradas, de color pardo rojizo, correspondientes a dos cérvidos, dos équidos y restos de un cuadrúpedo indeterminado.

A pesar de estar representados zoomortos diferentes, todos poseen una morfologia similar, tan solo diferenciada por la presencia de las cuernas. Por este motivo y por el caracter imcompleto de la mayoría de las figuras, sólo hemos analizado, como representativo de la totalidad, el équipo central del conjunto. Sus resultados han sido los siguientes:

a) Resultados de la aplicación de la Escala de Naturalismo:

1. Zoomorfo ....4,5 puntos ...... Estilo seminaturalista

b) Análisis de los convencionalismos formales:

Observando todas las figuras podemos señalar los siguientes rasgos característicos:

- Cabeza con apariencia real.

- Linea cérvico-dorsal sinuosa (dos figs.) o convexo-cóncava. 


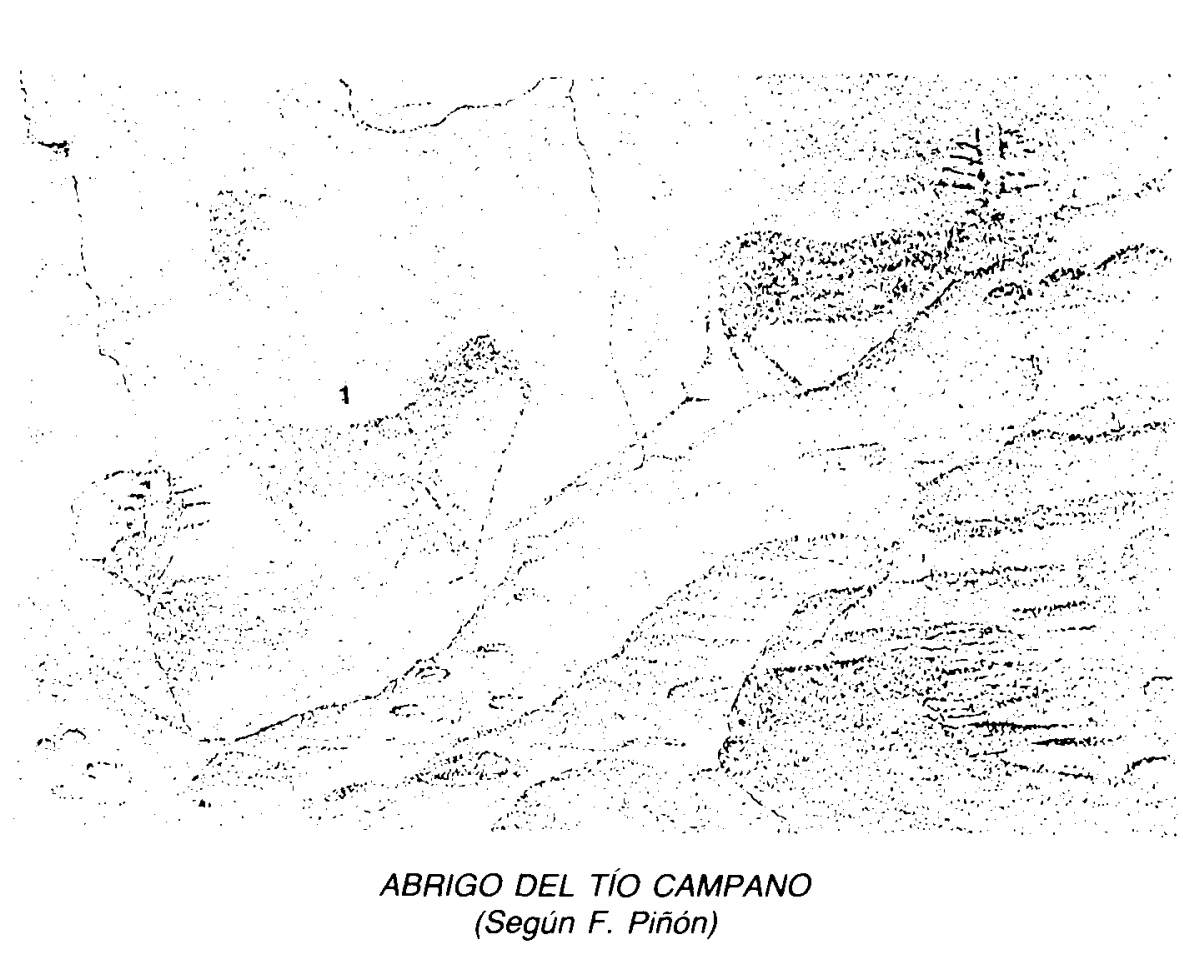

- Extremidades cortas, delgadas, rigidas, proyectadas hacia adelante y sin indicación de las pezuñas o las articulaciones.

- Rabo delgado.

- Cuerna de los cérvidos en perspectiva torcida, con las ramas ascendentes verticales y paralelas y los candiles perpendiculares a ellas y paralelos entre sí.

- Presencia en los équidos de unos objetos de fino trazado en prolongación del hocico que se bifurcan en el otro extremo.

Tal y como F. Piñón indicaba, estas figuras poseen una actitud de oposición contra el objeto citado, manifestada por la proyección hacia adelante de las extremidades, por la tensión del rabo y por la posición erguida de la cabeza (PIÑón, 1983, pág. 378).

La unidad del conjunto es evidente, perteneciendo todas las figuras a una misma fase. 
Análisis estilístico de los conjuntos del "Canjorro de Peñarrubia", ...

EL CONJUNTO DE “SELVA PASCUALA»

Las pinturas de este yacimiento, ubicadas en Villar del Humo (Cuenca), pero en una zona adyacente con el núcleo de Albarracín, fueron descubiertas en 1918 por E. HERNANDEZ-PACHECO (1959, págs. 430-431), aunque fue $\mathrm{H}$. Breuil quien realizó su primer estudio, pero presentando las figuras dispuestas al revés de como están en la realidad (BreulL, 1935, vol. IV, págs. 71-72, fig. 34).

Componen este grupo un total de seis figuras: dos équidos y un zoomorfo indefinido situado en la misma vertical, un antropomorfo, otro équido del mismo estilo que los anteriores y una barra con lóbulo inferior.

Los dos équidos de la izquierda han sido realizados sobre otras figuras anteriores, de las que todavia se pueden apreciar el rabo y parte del lomo y de sus cabezas, detalle este que ha pasado normalmente desapercibido. Pero lo que más llama la atención de este conjunto, es la asociación del antropomorfo con el équido mediante una fina linea, que para los primeros investigadores citados era el ronzal típico de las escenas de domesticación ya estudiadas del "Canjorro de Peñarrubia", pero que tras un estudio minucioso del profesor A. Beltrán, se llegó a la conclusión de que se trataba de una escena de caza con lazo (A. BELTRÁN, 1968a, pág. 81 , y 1968b, págs. 154-161).

Como figuras representativas del conjunto, hemos elegido para su análisis el antropomorfo, el équido de la derecha y el zoomorfo indefinido. Los resultados han sido los siguientes:

a) Resultados de la aplicación de la Escala de Naturalismo:

1. Antropomorfo ....2'5 puntos .....Estilo semiesquemático

2. Zoomorfo (équido) 6 '75 puntos .....Estilo naturalista

3. Zoomorfo ........2,5 puntos .....Estilo semiesquemático

b) Análisis de los convencionalismos formales:

El único antropomorfo existente en el grupo, debe su calificación semiesquemática a los detalles, apenas esbozados, de la cabeza manos y pies. Por lo demás, su mofología responde exactamente al tipo de figura esquemática en "doble $Y$ ", tan abundante en el Alto Guadalquivir y en el núcleo de Moclin (Granada), así como en otros núcleos del área esquemática. Hay que resaltar, respecto al antropomorfo que aqui estudiamos, que su morfología es un detalle más de realismo, por cuanto 


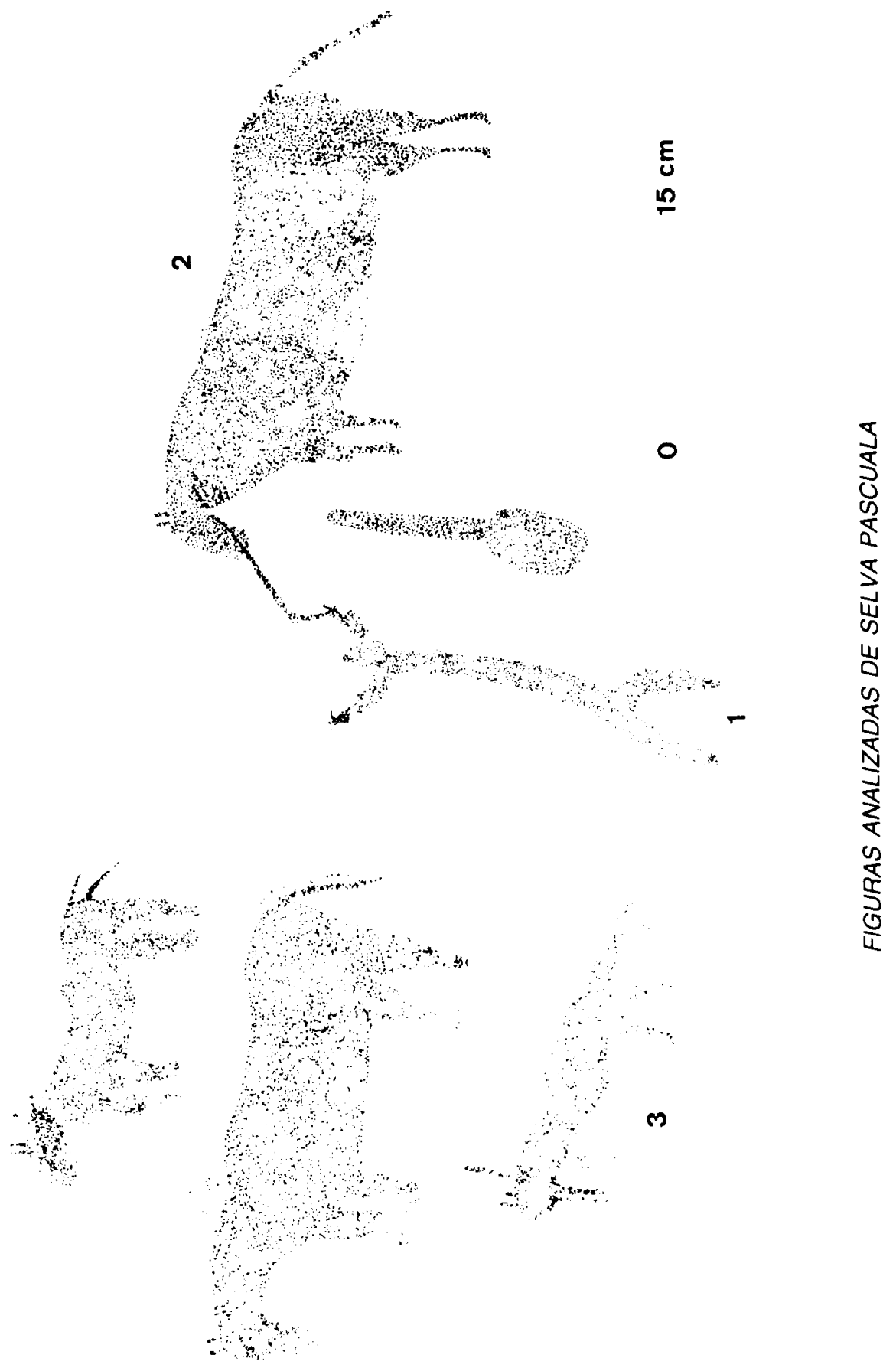


Análisis estilístico de los conjuntos del "Canjorro de Peñarrubia", ...

supone una adecuación de la forma a la acción de enlazar al équido por el cuello, lo que viene también a subrayarse por la cierta flexibilidad que se percibe en el cuerpo de la figura.

Respecto a los tres équidos naturalistas, poseen unos convencionalismos muy homogéneos:

- Cabeza alargada con apariencia casi real.

- Orejas pequeñas formadas por dos tracitos paralelos.

- Línea cervico-dorsal en triple curvatura.

- Tronco muy grueso, con vientre recto o ligeramente convexo.

- Rabo fino.

- Extremidades de aspecto desigual, aunque estan indicadas las pezuñas y se estrechan hacia abajo. No se indican las articulaciones.

El zoomorfo semiesquemático posee unos convencionalismos totalmente distintos a los anteriores, mientras que la barra con lóbulo inferior es un simbolismo propio del estilo esquemático.

A pesar de los detalles de repintado o superposición que en un principio indicamos, y de los resultados obtenidos al aplicar la escala de naturalismo, el cromatismo y la disposición de las figuras apoyan la idea de que todo el conjunto responda a una unidad compositiva. Lo poco que se observa de los primitivos zoomortos sobre los que se ejecutaron los dos équidos de la izquierda, parece indicarnos, por la semejanza de los rabos, que pudieran también tratarse de équidos muy similares a los que sobre ellos se realizaron.

\section{CONCLUSIONES}

Al efectuar un estudio comparativo de los resultados del análisis estilístico de estos conjuntos, hemos obtenido una serie de conclusiones que, en parte, habian sido brevemente esbozadas por otros investigadores y por nosotros, pero que gracias a nuestro método, se pueden concretar aún más e incluso añadir otras no menos importantes.

Así por ejemplo, en el caso de los conjuntos del "Canjorro de Peñarrubia" y de "Doña Clotilde», ya se había advertido su similitud en la 
forma de los équidos, en el aspecto globuloso de algunos antropomorfos y en la unión antropomorfo-équido por medio de la brida. Sin embargo, tras el análisis efectuado, podemos enumerar las siguientes semejanzas:

1. ${ }^{\text {a. }}$ Respecto a los antropomorfos, se dan en ambos conjuntos tres agrupaciones similares: En primer lugar observamos la presencia de antropomorfos simples de estilo esquemático (utípicos simples" en el "Canjorro de Peñarrubia" y "ancoriformes" en "Doña Clotilde"); en segundo lugar, también aparecen en ambos conjuntos arqueros esquemáticos de fino trazado y cabeza aplanada con arcos de una sola curvatura; y finalmente, también están presentes los antropomorfos de aspecto globuloso.

2. ${ }^{\text {a }}$ En ambos conjuntos son los antropomorfos de aspecto globuloso los que estan asociados a los équidos.

$3 .^{a} \quad$ Esos mismos antropomorfos poseen idéntica puntuación en la escala de naturalismo (4 puntos) y, como rasgos comunes, una cabeza redondeada, cintura apenas esbozada para diferenciar el tórax del abdomen, y extremidades de fino trazado sin expresión de las manos ni de los pies.

$4 .^{\text {a }}$ Los zoomorfos mejor conservados de ambos conjuntos también poseen una puntuación similar en la escala de naturalismo $(4,75$ y 5 puntos). A esta circunstancia se añaden ciertos rasgos comunes, como son la presencia de cabeza con cierta apariencia real, representación de las orejas y unas extremidades rigidas, en las que no figuran ni pezuñas ni articulaciones. Por último, una fina línea representa el ronzal, que une la mano del antropomorfo con el hocico del animal.

Aunque el conjunto del "Tio Campano" solo posee figuras zoomorfas, su puntuación es también similar a los anteriores (4,5 puntos) y poseen ciertos rasgos comunes con el zoomorfo analizado de «Doña Clotilde». Entre ellos podemos citar los siguientes: cabeza con apariencia real, rabo fino y extremidades rígidas sin pezuñas ni articulaciones. La inserción hocico-ronzal de "Doña Clotilde" es similar a la del hocicoobjeto del «Tio Campano».

De todos los conjuntos analizados, es el de "Selva Pascuala" el que difiere notablemente del resto. Así se observa tanto en la puntuación de los équidos (6,75 puntos), como en sus convencionalismos formales, exceptuando el fino trazado del rabo. Igual ocurre con el antropomorfo (2,5 puntos). La única similitud entre este conjunto y los anteriores sólo puede 
formularse a un nivel más general e inconcreto, por cuanto en todos los casos se trataría del empleo de recursos estilísticos naturalistas para expresar escenas que asocian generalmente antropomorfos con équidos, $y$ que se refieren a acciones que permiten la manipulación del animal vivo, ya sean de domesticación o de caza con lazo u otros objetos.

Ante esta acumulación de datos y observaciones, no podemos por menos que afirmar, que existe una estrecha vinculación estilo-temática manifestada en determinados conjuntos, situados algunos de ellos geográficamente próximos, e incluso que esta vinculación es aún más fuerte en abrigos tan lejanos con el de "Doña Clotilde" y el "Canjorro de Peñarrubia", donde las similitudes son de tal calibre, que se podria lanzar la hipótesis de una relación directa explicable tal vez como producto de migraciones de tipo estacional.

Bajo estas premisas, la capacidad narrativa de este tipo de escenas no debe ser explicada como fruto del azar o del deseo momentáneo de sus autores, sino que además debe ser interpretada como producto de un sustrato pictórico existente principalmente en el área levantina, que se manifiesta con rasgos como los indicados, pero que suponen una total ruptura con las figuras de las fases clásicas del Estilo Levantino, hasta el punto de que este tipo de figuras no deben considerarse como pertenecientes a ese estilo. Esta observación, que ya fue efectuada en su momento por el profesor A. Beltrán (1982, págs. 32, 44 y 62), no ha sido suficiente para excluir el estudio de los conjuntos aqui analizados de las monografias dedicadas al Estilo Levantino o al Esquemático.

Nos encontramos por lo tanto con unos conjuntos que no son de Estilo Levantino ni Esquemático, a los cuales, por el momento podemos asignarles los calificativos de seminaturalistas y postpaleolíticos, términos todavia muy genéricos que habrá que concretar en el futuro.

Finalmente, queda aún otro tema por abordar y es el relativo a la cronología de estos grupos y su posición relativa respecto a los estilos citados.

Las primeras opiniones situaban estos conjuntos en el punto medio de la línea evolutiva que conducía del Estilo Levantino al Esquemático, y cuya realización era explicada como producto de una "evolución artística degenerativa" del Estilo Levantino, que se concretaría al final del Mesolítico o principios del Neolítico (Hernández-PACHECO, 1959, pág. 414). Opinión que en líneas generales también defendia BOSCH GIMPERA (1965, págs. 1-12). 
Las investigaciones posteriores han coincidido en señalar una cronología más reciente para estos conjuntos, pero no han coincidido en su adscripción estilística.

Así por ejemplo, el profesor Almagro Basch habla de una perduración del naturalismo en una época en que en otras regiones ya se habia implantado el esquematismo. También indica que los équidos domesticados sean posiblemente asnos (ALMAGRO BASCH, 1963, págs. 460, 461 y 469).

Por su parte el profesor E. Ripoll, haciéndose eco de las caracteristicas seminaturalistas de las figuras de "Selva Pascuala", las situa en la fase final del Estilo Levantino, mientras que las del "Canjorro de Peñarrubia» y "Doña Clotilde" pertenecerian a la fase $D$ o de transición al Arte Esquemático (RIPOLL, 1966, págs. 176-178).

El profesor A. Beltrán, que no admite el carácter levantino de estas figuras y que defiende una ruptura entre el Estilo Levantino y el Esquemático, situa cronológicamente estos conjuntos en una fase final no levantina posterior al 2000 a.C., dentro de una época esquemática, indicando el detalle de que los équidos no aparecen montados y coincidiendo con Almagro en que podian ser asnos (BELTRÁN, 1982, págs. 32, 44, 62 y 63).

En lo que respecta al abrigo del "Tío Campano", fue situado cronológicamente por su investigador en un momento anterior a la escena de domesticación de "Doña Clotilde» (F. PINÓN, 1983, pág. 382).

La aportación de los datos arqueológicos para clarificar el problema cronológico es aún muy insuficiente. Ciñéndonos a los abrigos estudiados, sólo podemos aportar la noticia dada por Breuil sobre el hallazgo de cerámicas de aspecto eneolítico encontradas en el suelo del abrigo del "Canjorro de Peñarrubia" (BreUIL, 1933, vol. III, pag. 49) y los hallazgos de industrias mesolíticas y neoliticas efectuados por el profesor Almagro en el abrigo de «Doña Clotilde», que él calificó culturalmente como pertenecientes al Eneolitico y al Bronce (AlMAGRo BASCH, 1963, pág. 460).

Las excavaciones arqueológicas tampoco son muy relevantes en cuanto al estudio de la domesticación de équidos. Todavía son muy inconcretos los datos referentes a una posible domesticación neolítica de équidos, situándose los hallazgos de caballo doméstico de Los Castillejos de Montefrio (Granada) en la transición Neolitico-Calcolitico (ARRIBAS y MolinA, 1978, págs. 152-168), aunque persiste el problema del agiotipo anterior. 
Todo parece indicar, a pesar de la parquedad de las aportaciones arqueológicas, como asi lo expresa el profesor E. Ripoll, que figuras y escenas como las representadas en el "Canjorro de Peñarrubia", podrian tener una cronología entre el 3000 y el 2500 a.C. (RIFOLL, 1966, pág. 178), lo que ha venido a ser confirmado por los datos procedentes de las excavaciones de Los Castillejos.

Esta fechación podría también situarse en una fecha algo anterior si así lo vinieran a confirmar los hallazgos arqueológicos. Lo que no compartimos es la idea, al menos para el Sureste, de que este tipo de escenas se realizaran en una fecha posterior al 2000 a.C., dado que es a partir de ella cuando se desarrolla la Cultura del Argar, con la cual no creemos relacionadas las pinturas.

El marco cronológico citado guardaría concordancia con la persistencia de un sustrato de carácter narrativo una vez finalizadas las fases clásicas del Estilo Levantino, lo que pudo suceder a finales del IV milenio a.C., o principios del III milenio a.C., momento en el que se generaliza la economía de producción.

Otro problema por resolver es saber si el tipo de figuras aquí estudiado, pudo llevar, mediante un proceso de simplificación, al Estilo Esquemático propiamente dicho. Los datos que estos mismos abrigos proporcionan pueden ser usados en uno u otro sentido, ya que, por un lado, aparecen figuras esquemáticas con posibilidad de ser anteriores a las seminaturalistas (superposición de "Doña Clotilde" según Piñón); otras, por su situación fuera de la zona central del conjunto, pueden ser posteriores (véanse los antropomorfos esquemáticos del «Canjorro de Peñarrubia"); y otras podrían, ellas mismas, ser el preludio de tipos netamente esquemáticos, como es el caso del antropomorfo de "Selva Pascuala".

Para profundizar en la resolución de este problema, habria que hacer un análisis estilístico-tipológico muy exhaustivo de las figuras de las fases finales del Estilo Levantino y estudiar los posibles procesos de simplificación que en ellas se hubieran podido producir. Aún así, somos conscientes de las grandes dificultades con que este estudio contaría, pues siempre será difícil delimitar y distinguir un proceso de simplificación producido en la propia dinámica evolutiva de un estilo como el Levantino, de otro proceso de simplificación evidenciado en esas mismas figuras, pero que hubiera sido producido por influencias culturales procedentes de las poblaciones cuyo estilo fuera el Esquemático. 


\section{BIBLIOGRAFIA}

Acosta, P. (1983), “Técnicas, estilo, temática y tipología en la pintura rupestre esquemática hispana", Actas del coloquio internacional sobre Arte Esquemático de la Península Ibérica, Salamanca 1982, Zephyrus, XXXVI, Salamanca, págs. 13-25.

ALmagro BASCH, M. (1944), "Los problemas del Epipaleolítico y Mesolitico en España", Ampurias, VI, Barcelona, págs. 1-38.

ALmagro BASCH, M. (1949), "Un nuevo grupo de pinturas rupestres en Albarracín: La cueva de Doña Clotilde», Teruel, I, 2, Teruel, págs. 90-116.

Almagro Basch, M. (1963), "El Arte Rupestre Naturalista del Levante Español y el Arte Rupestre Esquemático", Historia de España de R. Menéndez Pidal, vol I, Ed. Espasa Calpe, Madrid.

Arribas, A., y Molina, F. (1978), «El poblado de Los Castillejos en las Peñas de los Gitanos (Montefrío, Granada), Campaña de excavaciones de 1971, El corte, núm. 1", Cuadernos de Prehistoria de la Universidad de Granada, Serie Monográfica, 3, págs. 152-168.

Beltrán Martínez, A. (1968a), "Sobre la pintura rupestre levantina de un caballo cazado a lazo del abrigo de Selva Pascuala en Villar del Humo (Cuenca)", Miscelánea Lacarra, Zaragoza, págs. 81 y ss.

Beltrán Martinez, A. (1968 b), Arte Rupestre Levantino, Seminario de Prehistoria y Protohistoria, Facultad de Filosofia y Letras, Monografias Arqueológicas, IV, Zaragoza.

Beltran Martinez, A. (1982), De cazadores a pastores. El Arte Rupestre del Levante Español, Col. Las Huellas del Hombre, Ediciones Encuentro, Madrid.

Beltrán Martinez, A. (1986), “El Arte Rupestre en la provincia de Teruel», Cartillas Turolenses, Instituto de Estudios Turolenses, Teruel. 
Análisis estilístico de los conjuntos del "Canjorro de Peñarrubia", ...

Beltrán Martínez, A. (1989), Ensayo sobre el origen y significación del Arte Prehistórico, Universidad de Zaragoza, Zaragoza.

Bosch GIMPERA, P. (1965), “La chronologie de l'art rupestre seminaturaliste et schématique et la culture megalithique portugaise", Revista da Facultade de Letras, núm. 9, Lisboa.

BreuIL, H. (1933), Les peintures rupestres schématiques de la Péninsule Ibérique, vol III, Sierra Morena, Lagny.

Breuil, H. (1935), Les peintures rupestres schématiques de la Péninsule Ibérique, vol IV, Sud-est et est de l'Éspagne, Lagny.

Hernández-Pacheco, E. (1959), Prehistoria del Solar Hispano. Origenes del Arte Pictórico, Memorias de la Real Academia de Ciencias Exactas, Fisicas y Naturales de Madrid, Serie de Ciencias Naturales, tomo $X X$, Madrid.

LÓpez PAYeR, M. G. y SORIA Lerma, M. (1988), El Arte Rupestre en Sierra Morena Oriental, La Carolina (Jaén).

López PAyer, M. G. y Soria LeRma, M. (1991), "Hacia una nueva metodología de la investigación del Arte Rupestre Postpaleolítico en Andalucía.-El análisis estilístico", Actas del // Congreso Internacional "El Estrecho de Gibraltar", UNED, Ceuta 1990 (en prensa).

Pinón VARela, F. (1982), Las pinturas rupestres de Albarracín (Teruel), Centro de Investigaciones y Museo de Altamira, Monografías núm. 6 , Santander.

Pinón Varela, F. (1983), "El Abrigo del Tío Campano (Albarracín, Teruel)", Homenaje al prof. Martin Almagro Basch, Ministerio de Cultura, Madrid, págs. 371-384.

Ripoll Perello, E. (1968), "Cuestiones en torno a la cronología del arte rupestre postpaleolítico en la Península Ibérica», Simposio Internacional de Arte Rupestre, Barcelona 1966, Barcelona, págs. 165-192.

Ripoll Perello, E. (1983), "Cronologia y periodización del esquematismo prehistórico en la Península Ibérica", Actas del Coloquio Internacional sobre Arte Esquemático de la Peninsula Ibérica, Salamanca 1982, Zephyrus, XXXVI, Salamanca, págs. 28-35.

Soria Lerma, M., y Lopez PAyer, M. G. (1989), El Arte Rupestre en el Sureste de la Península lbérica, La Carolina (Jaén).

Tamain, G. (1962), «Pinturas rupestres inéditas del «Barranco de la Moneda" en Baños de la Encina (Jaén)", Oretania, 11, mayo-agosto 1962, Linares, págs. 220-229.

Tamaín, G. (1963), "Los Canforos de Peñarrubia. El Centenillo (Jaén), Espagne. Nouvelle analyse des peintures rupestres", Bulletin de la Société Préhistorique Française, LX, págs. 828-837. 